\title{
Minor postoperative morbidity should not be neglected
}

\author{
Amedeo Anselmi, MD, PhD, and Erwan Flécher, MD, PhD \\ From the Division of Thoracic and Cardiovascular Surgery, Pontchaillou University Hospital, Rennes, France. \\ Disclosures: Authors have nothing to disclose with regard to commercial support. \\ Received for publication April 11, 2016; accepted for publication April 11, 2016; available ahead of print May 18, \\ 2016. \\ Address for reprints: Amedeo Anselmi, MD, PhD, Division of Thoracic and Cardiovascular Surgery, Pontchaillou \\ University Hospital, 2, rue Henri Le Guilloux, 35033 Rennes, France (E-mail: amedeo.anselmi@alice.it). \\ J Thorac Cardiovasc Surg 2016;152:602 \\ $0022-5223 / \$ 36.00$ \\ Copyright (C) 2016 by The American Association for Thoracic Surgery \\ http://dx.doi.org/10.1016/j.jtcvs.2016.04.038
}

In the era of the Placement of AoRTic TraNscathetER Valves II (PARTNER II) and SUrgical Replacement and Transcatheter Aortic Valve Implantation (SURTAVI) trials, cardiac surgeons realize once more that even minor postoperative adverse events - and their impact on the promptness of postoperative recovery and return to active lifestyle-are part of the cause in the comparative evaluation of established, effective, and durable surgical strategies versus less-perfected yet less-invasive techniques for the treatment of heart diseases. Appropriately, the current article by Balzer et al ${ }^{1}$ ultimately underlines that much improvement can still be done in the prevention of early postoperative morbidity associated with the accumulation of shed blood into the chest and pericardial cavities after cardiac surgery, which leads to repeat or prolonged hospitalization, additional treatments, increased costs, and delayed recovery. The authors need to be commended for this perspective; nonetheless, this paper needs to be attentively interpreted, because several different entities (reoperation for bleeding, pericardial drainage for tamponade and delayed thoracentesis/chest tube insertion for pleural effusion, independently from the demonstration of the hematic nature of the effusion) uniformly concur to the definition of the retained blood syndrome, and no stratified analysis has been performed.

As Balzer et al exhaustively point out, the daily practice of drain management in the intensive care unit is unsupported globally by any evidence. Each institution and often each surgeon have developed its/his own protocols through long-term experience and common sense. Beyond this, one must acknowledge that actually we do not definitively know the answer to basic questions associated with our daily activity (which drain diameter might optimize results for either the pericardial or pleural cavities; should the drains be milked, stripped, or not manipulated at all; which type of drain would perform better). ${ }^{2}$

For the aforementioned reasons, we believe that such lack of evidence should not be neglected. Patient satisfaction derives from the quality and rapidity of postoperative recovery

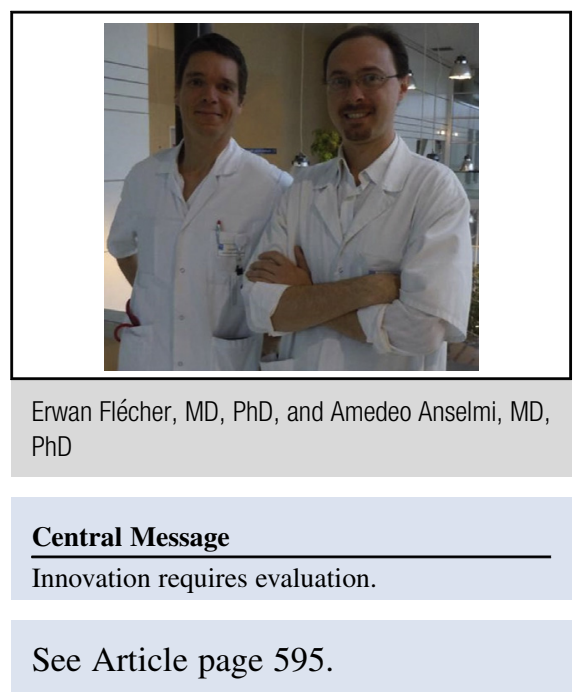

no less than from the effectiveness of the surgical repair of heart disease, mainly for elective cases and patients with limited preoperative symptoms. In such perspective, our Institution is supporting a multicenter national French trial aimed at establishing the usefulness of drain manipulation in the intensive care unit after cardiac surgery.

In their Perspective statement, the authors suggest that novel technologies for active drain clearing may be useful for the reduction of the incidence of retained blood syndrome. Everyone will today agree that any improvement in postoperative care is a significant determinant of the outcome after cardiac surgery, maybe as significant as the effectiveness of the operation for which the patient has been referred. Nonetheless, and as a reasonable consequence, even in this domain, any advances (use of new devices for drain clearance) must be accompanied by a rigorous appraisal of benefits and of the reasonable application for this technology before it enters routine practice. Dedicated trials and publication of evidence-based criteria are required in this respect. The more they will be methodologically sound, the more solid the background for the diffusion of the new technology will be. As for all other domains of cardiothoracic surgical research, innovation means evaluation.

\section{References}

1. Balzer F, von Heymann C, Boyler E, Wernecke K, Grubitzsch H, Sander M Impact of retained blood requiring reintervention on outcomes after cardiac surgery. J Thorac Cardiovasc Surg. 2016;152:595-601.

2. Wallen M, Morrison A, Gillies D, O'Riordan E, Bridge C, Stoddart F. Mediastinal chest drain clearance for cardiac surgery. Cochrane Database Syst Rev 2004;4 CD003042. 